\title{
The novel role of paramedics in collaborative emergency centres aligns with their professional identity: A qualitative analysis
}

\author{
Stewart Whalen, $\mathrm{BSc}^{*}{ }^{\dagger}$; Judah Goldstein, $\mathrm{PCP}, \mathrm{PhD}^{\dagger \S \pi}$; Robin Urquhart, $\mathrm{PhD}^{\ddagger \pi}$; \\ Alix J.E. Carter, MD, MPH ${ }^{\dagger \S \Phi}$
}

\section{CLINICIAN'S CAPSULE}

What is known about the topic?

Little is known about the experiences of paramedics working in CECs.

What did this study ask?

This study sought to ascertain the attitudes, feelings and experiences of paramedics working within the Nova Scotia CEC construct.

What did this study find?

Positive collaboration between RNs and paramedics, need for improved support from leadership, value placed on CECs by communities and this new role aligning with paramedic professional identity.

Why does this study matter to clinicians?

This study will contribute to the ongoing growth of the model by providing clinicians and decision makers with valuable information from frontline workers on CEC functioning.

\section{ABSTRACT}

Objective: The Collaborative Emergency Centre (CEC) model of care was implemented in Nova Scotia without an identifiable, directly comparable precedent. It features interprofessional teams working towards the goal of providing improved access to primary health care, and appropriate access to 24/7 emergency care. One important component of CEC functioning is overnight staffing by a paramedic and registered nurse (RN) team consulting with an off-site physician. Our objective was to ascertain the attitudes, feelings and experiences of paramedics working within Nova Scotia's CECs.

Methods: We conducted a qualitative study informed by the principles of grounded theory. Semi-structured telephone interviews were conducted with paramedics with experience working in a CEC. Analysis involved an inductive grounded approach using constant comparative analysis. Data collection and analysis continued until thematic saturation was reached. Results: Fourteen paramedics participated in the study. The majority were male $(n=10,71 \%)$ with a mean age of 44 years and mean paramedic experience of 14 years. Four major themes were identified: 1) interprofessional relationships, 2) leadership support, 3) value to community and 4) paramedic identity.

Conclusions: Paramedics report largely positive interprofessional relationships in Nova Scotia's CECs. They expressed enjoyment working in these centres and believe this work aligns with their professional identity. High levels of patient and community satisfaction were reported. Paramedics believe future expansion of the model would benefit from development of continuing education and improved communication between leadership and front-line workers.

\section{RÉSUMÉ}

Introduction: Les centres d'urgence collaborative (CUC) est un modèle de soins sans pareil qui a été mis en place en Nouvelle-Écosse. II fait intervenir des équipes interprofessionnelles dans le but d'améliorer l'accès aux soins primaires et de permettre un accès approprié à des soins d'urgence, $24 \mathrm{~h}$ sur 24, 7 jours sur 7 . Un élément important du fonctionnement des CUC est la formation d'équipes de nuit, composées d'infirmières et d'ambulanciers paramédicaux qui sont en consultation avec des médecins hors des lieux de l'événement. L'étude visait donc à sonder les attitudes, les sentiments et l'expérience des ambulanciers paramédicaux qui travaillent dans les CUC.

Méthode: II s'agit d'une étude qualitative, menée selon les principes de la théorie ancrée dans la pratique. Des entretiens semi-directifs téléphoniques ont été réalisés avec des ambulanciers paramédicaux ayant une expérience de travail dans les CUC. L'analyse a été effectuée selon une démarche inductive, enracinée dans la pratique, sur le mode de

From the *Faculty of Medicine, Dalhousie University, Halifax, NS; tDepartment of Emergency Medicine, Division of Emergency Medical Services, Dalhousie University, Halifax, NS; ‡Department of Surgery, Dalhousie University, Halifax, NS; §Emergency Health Services Nova Scotia, Dartmouth, NS; and; INova Scotia Health Authority, Halifax, NS.

Correspondence to: Stewart Whalen, Dalhousie Department of Emergency Medicine, Division of EMS, Halifax Infirmary, Room 3021,1796 Summer Street, Halifax, NS B3H 3A7; Email: stewart.whalen@dal.ca 
l'analyse comparative continue. La collecte de données et I'analyse se sont poursuivies jusqu'à saturation thématique.

Résultats: Quatorze ambulanciers paramédicaux ont participé à l'étude. La majorité était composée d'hommes $(n=10$; $71 \%)$; l'âge moyen s'élevait à 44 ans et les participants comptaient en moyenne 14 ans d'expérience en soins paramédicaux. Se sont dégagés de la collecte de données quatre grands thèmes : 1) les relations interprofessionnelles; 2) le soutien des autorités; 3) la valeur pour la communauté, et 4) la profession paramédicale.

Conclusion: Les ambulanciers paramédicaux ont fait état, en grande partie, de bonnes relations interprofessionnelles dans les CUC, en Nouvelle-Écosse. Ils ont exprimé du plaisir à travailler dans ces centres et ils croient que ce genre de travail va dans le même sens que les valeurs de la profession. Les patients et la communauté ont aussi indiqué un degré élevé de satisfaction. Toutefois, les ambulanciers paramédicaux sont d'avis que le développement futur du modèle profiterait d'un approfondissement de la formation continue et d'une amélioration des communications entre les autorités et les travailleurs de première ligne.

Keywords: Paramedic, Collaborative Emergency Centre, professional identity, EMS

\section{INTRODUCTION}

The Collaborative Emergency Centre (CEC) model of care was developed in Nova Scotia in response to limited access to primary care and frequent closures of rural emergency departments (EDs), reported to total 19,116 hours in the province between 2009 and 2010. ${ }^{1}$

A CEC has three components: 1) a primary health care team; 2) urgent care capacity; and 3) emergency care in collaboration with emergency health services (EHS) and the health authority. During extended daytime hours, an on-site physician oversees all aspects of care. Overnight urgent and emergency care is delivered by a paramedic and registered nurse ( $\mathrm{RN})$ team, consulting with an off-site physician by telephone. An evaluation by Stylus Consulting reported communities in which a CEC had been implemented experienced a $90 \%-100 \%$ reduction in hours of unplanned overnight ED closures since inception. ${ }^{2}$

Although the CEC design stems conceptually from other collaborative systems across Canada, there are few, if any, comparable examples of paramedics operating under a CEC-type paradigm. ${ }^{3,4}$ The purpose of this study was to ascertain the attitudes, feelings, and experiences of paramedics working in these novel centres.

\section{METHODS}

\section{Study design}

We conducted a qualitative study with semi-structured interviews of paramedics using data collection and analysis methods informed by grounded theory. ${ }^{5}$ The study was shaped by research into the expanded role of paramedics in Nova Scotia and emerging research into the CEC model. Ethics approval was obtained from the
Nova Scotia Health Authority Research Ethics Board (file \# 1020037).

\section{Participants}

Participants were a convenience sample of paramedics with experience working in a CEC in Nova Scotia. There were no other inclusion or exclusion criteria. The EHS registrar distributed an email invitation to all paramedics registered in the province, directing interested parties to contact the principal investigator (PI). The PI (SW) initiated follow-up with potential participants to ensure that they met the eligibility criteria before scheduling a time to conduct the interview.

\section{Data collection}

Semi-structured telephone interviews were completed between July 2015 and February 2016. The PI conducted all interviews. Interview questions were modified during data collection to ensure developing categories were explored.

\section{Analysis}

The analysis employed an inductive grounded approach with a constant comparative analysis. ${ }^{5}$ The first four interviews were coded and analyzed independently by all members of the research team after which a coding framework was developed to guide categorization. The PI coded and analyzed the remaining transcripts.

Open and axial coding of interview transcripts occurred simultaneously. Qualitative analysis was performed manually using Microsoft Word. Data collection and analysis continued until thematic saturation was reached..$^{5}$ The research team then met to develop categories and integrate these into major themes. 


\section{RESULTS}

Fourteen paramedics participated in the study. Participants were predominately male $(n=10 ; 71 \%)$, with a mean age of $43.6 \pm 8.8$ years and $14 \pm 9.7$ years of paramedic experience, of which $2.5 \pm 1.1$ years were spent working in CECs.

Four dominant themes were identified: 1) interprofessional relationships; 2) leadership support; 3) value to the community; and 4) paramedic identity.

\section{Interprofessional relationships}

The paramedic and $\mathrm{RN}$ working relationship was perceived to be difficult initially, with participants viewing RNs as unwelcoming (Table 1, quote 1a). Differences in culture and experience between the two professions were perceived to represent a further challenge to team functioning. For example, participants highlighted their ability to function independently and viewed RNs as uncomfortable doing the same (Table 1, quote 1b).

Despite these challenges, participants viewed their overall experience with RNs positively (Table 1, quote 1c, 1d). They perceived their relationship to be collaborative and strengthened over time through shared experience (Table 1, quotes 1e). Participants were overwhelmingly satisfied with off-site physician support (Table 1, quote 1f).

\section{Leadership support}

Participants viewed support from EHS operations, the health authority, and the government to be inadequate because of a lack of feedback and follow-up (Table 1, quote $2 \mathrm{a}, 2 \mathrm{~b}$ ), as well as absent communication on the long-term future of CECs (Table 1, quote 2c).

Participants expressed a desire to expand their scope of practice to include additional skills (Table 1, quote 2d, 2e) and identified continuing medical education (CME) tailored to CEC practice as an area of need (Table 1, quote $2 \mathrm{f}$ ).

\section{Value to community}

Participants viewed CECs as valuable to communities and patients. They perceived high levels of satisfaction from patients who receive care without travelling and waiting for long periods in larger EDs (Table 1, quote $3 \mathrm{a}, 3 \mathrm{~b}$ ).

\section{Paramedic identity}

Participants perceived working in CECs to be in line with their professional identity. They viewed this novel work as a continuation of the expanding role of paramedics in Nova Scotia (Table 1, quote 4a) and beneficial for the public perception of the profession (Table 1, quote 4b).

Participants found working in CECs to be both professionally and personally rewarding. Professional reasons included career diversity (Table 1, quote 4c), participation in the continuation of care, and skill acquisition (Table 1, quote $4 d$ ). Personal reasons included reducing travel, particularly in inclement weather (Table 1, quote 4e); not having to listen to dispatch communications; and opportunities to build relationships with community members.

\section{DISCUSSION}

Effective interprofessional teamwork is an increasingly essential component of quality patient care. ${ }^{6}$ To our knowledge, the CEC model is unique in how the paramedic and $\mathrm{RN}$ teams work in a fixed location with off-site physician support. Despite initial challenges, our participants reported positive collaboration with their RN colleagues, in line with other studies looking at nurse and paramedic teams. ${ }^{7,8}$

Organizational support has been identified as an important factor in interprofessional team functioning. ${ }^{9}$ Our participants viewed this support as lacking in a number of areas and highlighted CEC-specific CME and improved communication as important to CEC functioning, as well as the future advancement of the model.

The value placed on CECs by communities and high levels of perceived patient satisfaction are positive findings that support CECs as viable and patient centred. The paramedic profession is growing with paramedics acquiring new skills and being employed in novel areas of health care. ${ }^{10}$ Our participants viewed the CECs as a continuation of this growth and their work in these centres to be rewarding and in line with their professional identity.

\section{LIMITATIONS}

While we used methods informed by grounded theory, this study was exploratory in nature versus theory generating. Future research may use these findings to 
Table 1. Participant quotes supporting the following themes: 1) interprofessional relationships; 2) leadership support; 3 ) value to the community; and 4) paramedic identity

1) Interprofessional relationships

1a "You're going into their [RN's] place or their spot. . . It's kind of like when someone comes in the back of your ambulance. You kind of get territorial." Participant 13

$1 \mathrm{~b}$ "They are emerg[ency] nurses so they do know what to do but they are very hesitant to act without direction. Where I would rather act and do and then call the doc and say what I've done and this is my result." Participant 7

$1 c$ "Once you build a rapport and you find out what your strengths and weaknesses are then you work as a health care team." Participant 5 $1 \mathrm{~d}$ " ... we have a really good working relationship between the paramedics and the staff at the hospital, the RNs and even the doctors as well. .. . I feel comfortable working with the RN and their skill set and I believe they feel comfortable working with us and our skill set." Participant 14

$1 \mathrm{e}$ ". ... we've had to develop a team that [wasn't] on the same wavelength at the beginning. ... There was animosity with the nurses, the paramedics coming into the nursing hospital. That's been worked through. They've come to appreciate our skills and we've come to appreciate theirs." Participant 1

$1 f$ "I find that they [off-site physicians] leave lots of leeway to help make our own decisions. They give guidelines and support but they also take input from what we do. There is most definitely good support there." Participant 4

2) Leadership support

2a "There's not a lot of input I find and we don't get any feedback from anybody. It's just kind of status quo where everything's good." Participant 6

$2 \mathrm{~b}$ "I find it very difficult for management to understand that we were given the training, put in the hospital, and there was never any follow up with us." Participant 1

2c " . . this program was brought in by the NDP Government ... when we switched to the Liberal Government here in Nova Scotia we were kind of holding our breath saying, 'is this system going to stay?'" Participant 2

$2 d$ "The scope of practice is the same as what we do on the units. I think the scope needs to expand in the CEC model." Participant 9

$2 \mathrm{e}$ "[Suturing] is something I feel would be very useful for us ... honestly we see more lacerations than broken bones ever." Participant 14

$2 f$ "As far as continuing education ... there's none and there needs to be. . . I think after two and a half years there needs to be more training because you walked into it kind of not knowing, so the basics was great but once you know more about what's going on with the CEC model I believe the more education will be better." Participant 9

3) Value to the community

3a "They [patients] tell you right upfront they're happy when they leave. They're smiling when they leave. They got the information that they want and they feel they got the care they needed for that time frame and then it's followed up with other visits . . " Participant 4

$3 \mathrm{~b}$ "It gives the community a peace of mind that there is a facility for them to come to with the children or the elderly. They have a place to come, a place to start that they're comfortable with, or in an emergency situation. They're very thankful that we're there." Participant 1

4) Paramedic identity

4a "I like the expanded scope and the profession is expanding from the ambulance to a wider scope. So, I think the CEC ... for me fits into my identity as a paramedic. For me, it's important to have other options and to expand scope and relationships and expand the profession and I think the CECs are one way to do that." Participant 11

$4 \mathrm{~b}$ "I think it opens the eyes to the public, like they think 'oh we're not just ambulance drivers,' that we're actually health professionals." Participant 5

4c "Personally, it's extremely satisfying for me to do this. It's a change of pace, it keeps the job fresh to go out and deliver primary care as opposed to emergent care." Participant 12

$4 d$ " . . I can read a book maybe five or six times but to have the resource of an RN, especially with 20 plus years' experience, sometimes they have little tricks of the trade that you can learn from them and pick up." Participant 5

$4 \mathrm{e}$ "Sometimes [on ambulances] you have to go out in inclement weather; you could be at a car accident at the side of the road in the middle of a snowstorm at 3 a.m. But when you're working in the hospital patients are coming to you." Participant 2

build and expand theory related to paramedic identity and expanded roles in interprofessional care.

Paramedics with past or present CEC experience could participate in this study. However, our enrolled participants were limited to active CEC paramedics. Furthermore, we did not obtain the perspectives of RNs or physicians working within the CEC model. Their perspectives may conflict with those of our participants, and future research should investigate this matter.

\section{CONCLUSIONS}

Paramedics reported initial challenges but largely positive interprofessional relationships with $\mathrm{RNs}$ in the 
CECs in Nova Scotia, supporting the viability of these teams in this model. Paramedics enjoy working in this novel role and believe it aligns with their professional identity. High levels of patient and community satisfaction were reported. Participants believe the future expansion of the model in Nova Scotia and beyond would benefit from the development of CEC-specific $\mathrm{CME}$ and regular communication between leadership and front-line workers.

Acknowledgements: We would like to acknowledge our participants, without whom this project would not be possible. Tremendous support was provided by both the Dalhousie Department of Emergency Medicine and Division of EMS and EHS Nova Scotia.

Competing interests: The PI was the recipient of two summer studentship grants through the Dalhousie Medical Research Foundation (DMRF) and the Research in Medicine (RIM) program at Dalhousie University, Faculty of Medicine. This research received no further financial support. The authors (SW, JG, RU, and AC) have no financial or other conflicts related to this publication.

\section{REFERENCES}

1. Nova Scotia Department of Health. Better care sooner the plan to improve emergency care. Halifax: Nova Scotia Department of Health; 2010.

2. Stylus Consulting, Nova Scotia Department of Health, and Wellness, Canadian EL. Care right now: evaluating the
Collaborative Emergency Centre experience in Nova Scotia. Halifax: Nova Scotia Department of Health and Wellness; 2014.

3. Hayden JA, Killian L, Zygmunt A, et al. Methods of a multifaceted rapid knowledge synthesis project to inform the implementation of a new health service model: Collaborative Emergency Centres. Syst Rev 2015;4:7.

4. Hayden J, Nova Scotia Cochrane RC. Collaborative emergency centres: rapid knowledge synthesis. Halifax: Nova Scotia Cochrane Resource Centre; 2012.

5. Strauss AL. Basics of qualitative research: grounded theory procedures and techniques. Newbury Park: Sage Publications; 1990.

6. Deneckere S, Euwema M, Lodewijckx C, et al. Better interprofessional teamwork, higher level of organized care, and lower risk of burnout in acute health care teams using care pathways: a cluster randomized controlled trial. Med Care 2013;51(1):99-107.

7. Martin-Misener R, Downe-Wamboldt B, Cain E, Girouard M. Cost effectiveness and outcomes of a nurse practitionerparamedic-family physician model of care: the Long and Brier Islands study. Primary Health Care Research \& Development. Prim Health Care 2009;10(1):14-25.

8. Machen I, Dickinson A, Williams J, Widiatmoko D, Kendall S. Nurses and paramedics in partnership: perceptions of a new response to low-priority ambulance calls. Accid Emerg Nurs 2007;15(4):185-92.

9. Xyrichis A, Lowton K. What fosters or prevents interprofessional teamworking in primary and community care? A literature review. Int 7 Nurs Stud 2008;45(1):140-53.

10. Evans R, McGovern R, Birch J, Newbury-Birch D. Which extended paramedic skills are making an impact in emergency care and can be related to the UK paramedic system? A systematic review of the literature. Emerg Med $\mathcal{f}$ 2014; 31(7):594-603. 